Pract. oto-rhino-laryng. 1962;24:343

\title{
On Osteogenesis Imperfecta
}

G.A.

\author{
Hoogland
}

\section{Arnhem}

Author's address: Dr. G. A. Hoogland, Apeldoornseweg 67, Arnhem (Netherlands)

A case of van der Hoeve-de-Kleyn syndrome-blue eyes

brittle bones and otosclerosis-is described in which stapedectomy according to Shea was performed. A favourable hearing gain has been maintained for more than six months. Clear otosclerotic lesions were found on histologic examination of the removed footplate. In agreement with Simson Hall and others

otosclerosis is supposed to be a monosymptomatic manifestation of the osteo-blastic

insufficiency which underlies osteogenesis imperfecta. The vulnerability of the area surrounding the oval window may explain why this latent osteoblastic insufficiency often presents itself only as otosclerotic deafness. 